\title{
LA CAMPAÑA ELECTORAL DEL 2017 EN EL ECUADOR. UN ANÁLISIS DESDE LA PERSPECTIVA DEL MARKETING SENSORIAL
}

\author{
THE 2017 ELECTION CAMPAIGN IN ECUADOR. AN ANALYSIS FROM THE \\ PERSPECTIVE OF SENSORY MARKETING
}

\author{
Fernando Hallo Alvear \\ Universidad Internacional del Ecuador \\ rohalloal@uide.edu.ec \\ Rafael Camino Guaján \\ Universidad Tecnológica Equinoccial \\ cgrc5707@ute.edu.ec
}

RECIBIDO: 03 / 04 / 2017

APROBADO: 19 / 05 / 2017

\begin{abstract}
Resumen
El presente trabajo tiene como objetivo analizar la aplicación de las estrategias de Marketing Sensorial que fueron trabajadas por ocho binomios candidatos a la presidencia y vicepresidencia durante la campaña electoral de primera vuelta en las elecciones desarrolladas en el territorio ecuatoriano el 19 de febrero del año 2017. Con el equipo de investigación conformado por Pamela Vargas y Daniela Gallo, se indagó y analizó toda la información acerca del tema con el fin de concluir, dentro del campo político, cómo las diversas propuestas y acciones que realizaron durante el periodo de la campaña electoral tuvieron su impacto en los electores desde un punto de vista sensorial. De manera objetiva y técnica se estudió la forma en que cada candidato decidió aplicar estrategias que fueron percibidas por los electores por medio de sus sentidos, generando durante este periodo recordación u olvido, aceptación o rechazo, empatía o antipatía y en la mayoría de los casos, un voto. La estructura y enfoque del presente artículo es netamente técnico y divulgativo a nivel académico, no pretende dar juicios de valor ni posturas partidistas de ninguna índole a favor o en contra de los candidatos que formaron parte del estudio, así como tampoco se pretende concluir cuál estrategia fue mejor que otra o profundizar en temas políticos.
\end{abstract}

Palabras clave:

Marketing Sensorial, elecciones 2017, Marketing Emocional, percepción, sentidos.

\begin{abstract}
The present work aims to analyze the application of Sensory Marketing strategies that were worked by eight binomial candidates for the presidency and vice presidency during the election campaign of the first round in the elections held in Ecuador on February 19, 2017. With the research team made up of Pamela Vargas and Daniela Gallo, all the information about the subject was investigated and analyzed in order to conclude, within the political field, how the various proposals and actions carried out during the electoral campaign period its impact on voters from a sensory point of view. In an objective and technical way, it was studied how each candidate decided to apply strategies that were perceived by the voters through their senses, generating during this period remembrance or forgetfulness, acceptance or rejection, empathy or antipathy and in most cases, a vote. The structure and approach of this article is purely technical and informative at the academic level, it does not pretend to give value judgments or partisan
\end{abstract}


attitudes of any kind for or against the candidates that were part of the study, nor is it intended to conclude what strategy was better than another or to deepen in political subjects.

Key words:

Sensory Marketing, Elections 2017, Emotional Marketing, perception, senses

\section{Introducción}

Desde el aparecimiento en 1960 de la propuesta de Marketing Mix por Jerome McCarthy, pero que se popularizó en 1967 por Philip Kotler, hasta el momento toda la teoría del Marketing ha sufrido cambios obligados por las variaciones del entorno y del consumidor. Sin embargo, se mantiene la filosofía que es la de alcanzar la satisfacción de sus necesidades.

A partir de 1973, Kotler acuña el término atmospheric al control de variables que influyen en la respuesta del consumidor, provocando que el término evolucione reconociendo al marketing sensorial como ese conjunto de factores, variables y/o acciones que están controladas por el productor y distribuidor con el fin de crear una atmósfera denominada como multisensorial y que resulta específica en torno al producto dado, ya sea a través de sus características o del ambiente en el punto de venta (Filser, 2003).

Al analizar la perspectiva sensorial de la mente, parte fundamental de pasar del consiente al subconsciente y posteriormente al inconsciente al impulsar la generación de experiencias y delimitar momentos que se registren en la mente y emociones del consumidor, delimita el punto referencial del manejo del marketing sensorial también conocido como experiencial, al desarrollar atmósferas específicas. Estas generaciones de atmósferas definen el control intencional de variables ambientales con la finalidad de conseguir una determinada respuesta del consumidor (Turley, 2000).

Desde una perspectiva teórica es fundamental explicar la influencia de los factores sensoriales a través de los sentidos determinando los efectos en el consumidor, estos modelos se basan fundamentalmente en el modelo de Mehrabian y Russell (1974) donde determinan tres escalas dimensionales: placer, activación y dominancia.

Los modelos que parten de estos principios teóricos, así como del paradigma SOR (StimulusOrganism-Response) estímulo - organismo - respuesta, definen los efectos de las estrategias y acciones del marketing sensorial sobre las respuestas del consumidor, identificando dependencia de las emociones así como efectos motivacionales; es importante mencionar que la revisión bibliográfica señala resultados positivos, negativos e incluso también nulos referente al modelo SOR. Conociendo que el cerebro humano funciona de tal manera que la parte emocional y la parte racional se hallan siempre en una constante disputa por la retención de información, el momento que ésta la gana la parte emocional es cuando sobresalen los sentidos. Una forma de conseguir la diferenciación necesaria y que encaja con las necesidades de los consumidores, ha sido la creación de experiencias de compra. Estas experiencias donde se viven momentos diferentes y únicos dan lugar al denominado marketing experiencial o vivencial, y dentro de éste, al marketing sensorial. (Vieira, 2013)

El marketing sensorial participa directamente en la experiencia de compra en un punto de venta, utilizando para ello los cinco sentidos: vista, olfato, tacto, oído y gusto. (Manzano, Gavilán, \& Avelló, 2011) Al momento de generar contacto con los clientes se les debe ofrecer una experiencia sensorial sobresaliente que permita la correcta evaluación y selección de la alternativa en el proceso de decisión de compra del consumidor. Para esto es importante tocar como mínimo uno de los cinco sentidos de las personas y generar una reacción en cuanto a sensaciones y percepciones que sea satisfactorio. 
Vista

(Manzano, Gavilán, \& Avelló, 2011) expresan que la visión es el más poderoso de los cinco sentidos en el ser humano, ya que el $83 \%$ de la información que las personas retienen se recibe de manera visual en el cerebro. Al tener la visión esta importancia en los individuos, todo el contenido que ingresa por los ojos debe ser adecuado para conseguir resultados favorables.

Oído

Los seres humanos evaluamos todo lo que recibimos a través de los sentidos, pero es precisamente en el oído donde se decodifica más de 70\% de la información recibida (Lindstrom, 2007). La música es el principal estimulador para las emociones del ser humano y es por esto que las marcas la usan para varios propósitos por ejemplo influir en las sensaciones y conductas de las personas.

Tacto

Las sensaciones de la piel modifican los estados de ánimo, es por esta razón que el tacto es un factor muy importante que se debe tomar en cuenta en cualquier interacción comercial. "El tacto tiene una serie de características referenciales respecto a los demás sentidos, la primera de ellas es que implica un contacto directo, físico y sin intermediación con el producto, y es que este contacto se produce casi siempre a partir de una predisposición activa y voluntaria por parte del consumidor" (Manzano, Gavilán, \& Avelló, 2011).

Olfato

El olfato es uno de los sentidos que conecta la vida diaria y es uno de los más olvidados al momento de hacer publicidad, ya que las agencias descuidan o desconocen su fuerte influencia sobre el cerebro (Lindstrom, 2007). Según estudios varios el ser humano es capaz de recordar un $35 \%$ por el olor. Además, tardamos 10 años en olvidar un olor (Juan de Salinas, 2014).

Gusto

Junto con el olfato, el gusto es parte del sistema sensorial llamado químico, por el que los estímulos de este tipo provocan reacciones que activan los receptores responsables de su percepción. La principal diferencia entre ambos es que mientras en el sentido del olfato las moléculas que se captan son suficientemente pequeñas como para ser volátiles, en el caso del gusto, las moléculas no lo son, y se transmiten únicamente por contacto directo" (Manzano, Gavilán, \& Avelló, 2011). Indudablemente este sentido es el más completo, pues para que cualquier producto llegue a la boca es indispensable que primero pase por otros. (Pacheco, Carmona, \& Zapata, 2013) .

Este concepto se lo aplica también en una marca personal, pues se considera que cada persona es un producto y se debe comportar como tal para poder venderse. Desde el enfoque político, cada candidato se vuelve un producto que debe ser evaluado y aceptado por los votantes.

El objetivo de esta investigación es analizar la campaña electoral 2017 desde el enfoque de marketing sensorial, con el fin de evaluar cómo los binomios presidenciales lo aplicaron.

Según datos del Consejo Nacional Electoral CNE para las elecciones 2017, ocho binomios presidenciales fueron registrados:

Tabla 1

Candidatura a Binomios Presidenciales en las elecciones presidenciales del 2017

\begin{tabular}{ll}
\hline Organización Política & Binomio \\
\hline Creo-Suma & Guillermo Lasso y Andrés Páez \\
Acuerdo por el Cambio & Paco Moncayo y Monserrat Bustamante \\
Partido Fuerza Ecuador & Abdalá Bucaram y Ramiro Aguilar \\
Partido Social Cristiano & Cynthia Viteri y Mauricio Pozo \\
\hline
\end{tabular}




\begin{tabular}{ll}
\hline Movimiento Alianza País & Lenín Moreno y Jorge Glas \\
Movimiento Unión Ecuador & Washington Pesantez y Álex Alcívar \\
Movimiento Fuerza Compromiso Social & Iván Espinel y Doris Quiroz \\
Partido Sociedad Patriótica & Patricio Zuquilanda y Johnnie Jorgge \\
\hline
\end{tabular}

Fuente: (CNE, 2016)

Del listado presentado, se analizarán a cada uno de los binomios desde la perspectiva del Marketing Sensorial.

\section{METODOLOGÍA}

\section{Materiales y Métodos}

Para el presente documento se utilizó el método inductivo, el cual es un método científico que abarca conclusiones generales de cómo los candidatos a presidente y vicepresidente de la república organizaron sus campañas en torno al marketing sensorial con el propósito de llegar a ganarse los votos y la confianza de los ecuatorianos.

\section{Resultados}

Para presentar los resultados de la investigación, se enfocará el estudio sobre cada uno de los sentidos del ser humano que forman parte del Marketing Sensorial.

Vista

Muchos son los componentes que influyen visualmente al momento de presentar una marca. En el caso de las personas y su marca personal se toma en cuenta la comunicación no verbal, es decir, todo lo que la persona comunica de manera consciente o inconscientemente con el cuerpo. Incluyen factores como la gesticulación y los gestos.

Para analizar estos factores, en el caso de los candidatos se puede tomar en cuenta su forma de presentarse ante las personas de manera general y sin especificar en cada uno de los meetings o entrevistas.

Cynthia Viteri y Guillermo Lasso, utilizaron una correcta gesticulación al momento de dar sus conferencias demostrando confianza y seguridad. Su manera de vestirse es de forma casual en ambos casos, lo que denota una imagen de familiaridad.

Por otro lado, Abdalá Bucaram Pulley, mejor conocido como Dalo Bucaram, e Iván Espinel tuvieron una presentación más informal, buscando empatarse con el pueblo al igualar expectativas. Con Lenín Moreno, Paco Moncayo, Patricio Zuquilanda y Washington Pesántez por su lado, la imagen que manejaron fue más formal aparentando de seriedad y rigidez.

Uno de los ámbitos visuales a tomar en cuenta es el uso adecuado de los colores que identifican a una marca; en este caso, a un partido político. Los colores denotan significados especiales en determinados productos, ambientes o situaciones; el color y su poderoso aliado el diseño, connotan de forma conjunta significados que son percibidos por los consumidores de forma inmediata generando las primeras asociaciones a las marcas en la mente del consumidor (Manzano, Gavilán, \& Avelló, 2011).

Cada partido y movimiento político se identifica por sus colores, mismos que definen la transmisión sensorial en base al sentido de la vista para la retención del electorado. Estos permiten a la gente identificar a los distintos candidatos y sus propuestas de una manera simple y directa. 
Tomando en cuenta que cada color tiene distinto impacto sobre las personas de acuerdo a la sicología del color, se debe saber utilizarlos para causar el efecto deseado. Los candidatos escogieron los siguientes colores que representan a sus partidos políticos.

Tabla 2

Binomios presidenciales y sus logotipos partidistas

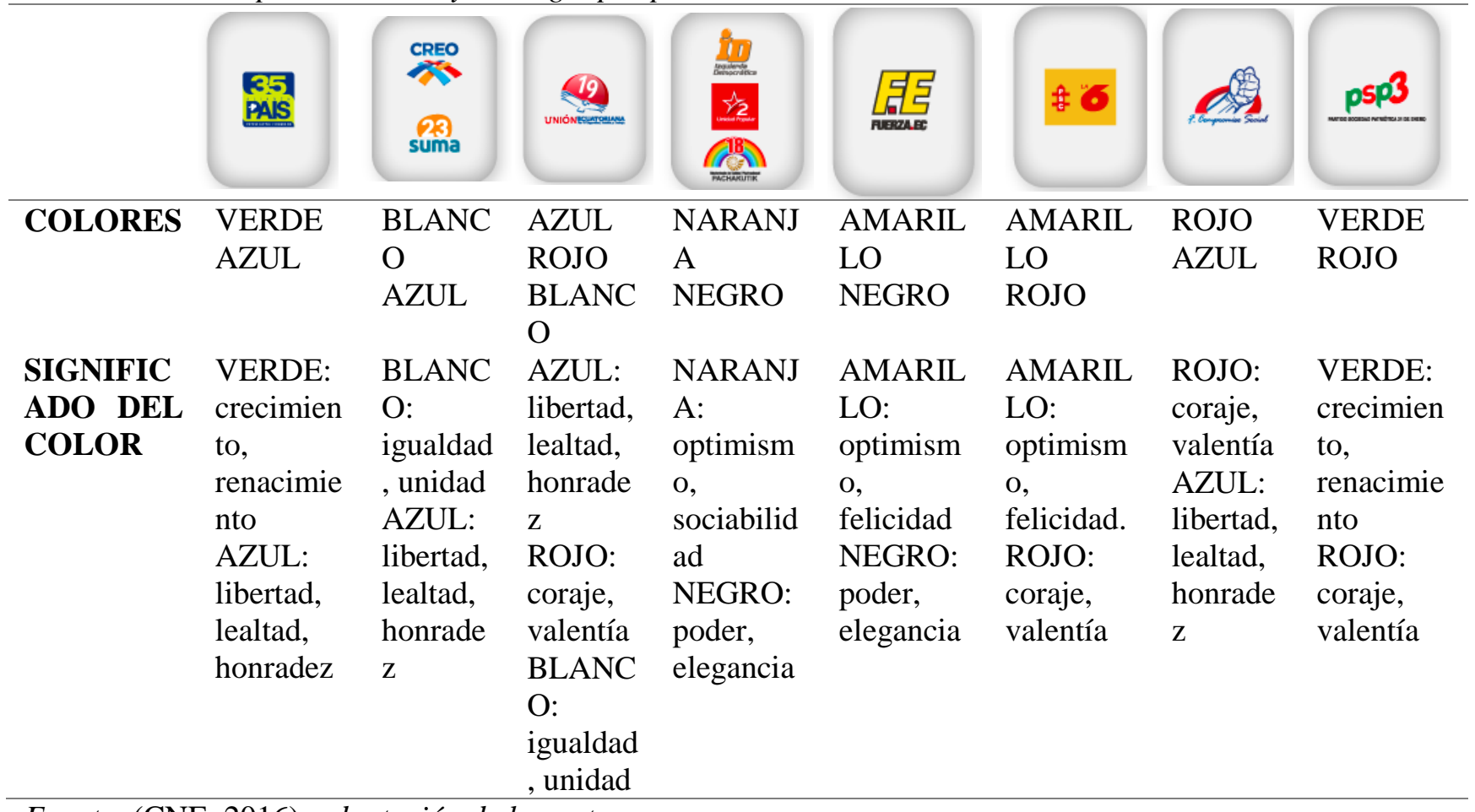

Fuente: (CNE, 2016), adaptación de los autores

Se evidencia que el color que sobresale es el azul debido a su representación de lealtad y honradez, prioritario para los ciudadanos. Los colores que toman en cuenta la mayoría de partidos se los asocia con la bandera del Ecuador, que transmite patriotismo y hace relación de sacar adelante al país.

El uso de los colores para las campañas electorales es vital ya que las ciudades se llenan con banderas, posters, flyers, publicidad móvil, etc. Esto permite visibilizar a los candidatos. Guillermo Lasso, por ejemplo, realizó activaciones BTL con dummies que lo imitaban físicamente de una manera muy llamativa.

Oído

En el caso de los candidatos, se usaron jingles en las redes sociales y emisoras del país que distinguieron a cada uno. En el caso de las redes sociales, no siempre fueron videos oficiales con canciones aprobadas por los partidos o movimientos políticos; sin embargo, existieron seguidores de los candidatos que realizaron estos videos con temas musicales para mantenerlos vigentes en la mente de los potenciales electores.

Como ejemplo están las canciones de: "Todo todito votamos 35" del movimiento político Alianza País, "Cambio Positivo" de Cynthia Viteri, "Salsa Choque 10 con 10" de Abdalá Bucaram, "Patricio Zuquilanda Presidente" de dicho candidato y "Un millón de empleos" de Guillermo Lasso. Estas canciones tuvieron miles de reproducciones en las redes sociales haciéndose presentes en plataformas virtuales de una manera más llamativa. 
Adicionalmente, es importante destacar el tono de voz que utilizaron los candidatos durante sus discursos, pues denotó mucho más que la emisión de un mensaje. La melodía de las palabras también permitió que los electores se inclinaran hacia uno u otro candidato. Moreno expresó sus ideas de una manera calmada y pausada, con poca gesticulación al igual que Guillermo Lasso y Paco Moncayo; por otro lado, Dalo Bucaram, Iván Espinel e incluso Cinthya Viteri manejaron un tono de voz alto y se comunicaron, en ciertos casos, gesticulando de manera fuerte. Patricio Zuquilanda y Washington Pesántez, manejaron discursos pausados pero firmes con un tono de voz cauto y constante.

\section{Tacto}

Debido a que los ecuatorianos son personas de cercanía, contacto y emociones, el tacto juega un papel primordial al momento en que una marca transmite un determinado mensaje. En este sentido se analiza el lenguaje proxémico que utilizaron los candidatos en las campañas políticas. La proxémica estudia la distancia que separa a dos personas cuando están interactuando (Bonnenmaison, 2017). Existen cuatro espacios creados para una interacción. La más extensa y alejada de nosotros que es el espacio público, una intermedia que es el espacio Socialconsultivo, el espacio personal-casual y el espacio íntimo. (Juan de Salinas, 2014)

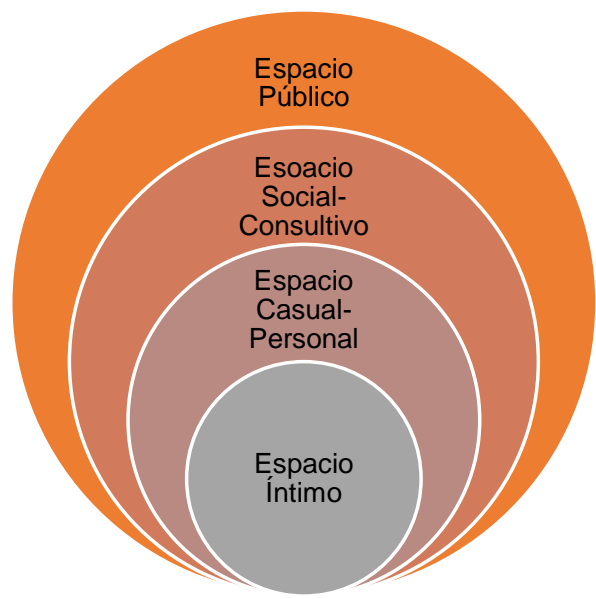

Figura 1. Proxémica, espacios referenciales. Obtenido de (Juan de Salinas, 2014)

El uso del tacto como estrategia política es fundamental en el acercamiento a los ciudadanos. Debido a la cultura ecuatoriana es muy común que los candidatos se acerquen a las personas a saludar, abrazar y besarlos. De esta forma se logra transmitir sinceridad, compromiso y familiaridad.

En todos los candidatos se evidenció el uso del tacto para comunicarse con las personas, algunos hicieron más uso de esto que otros. Dalo Bucaram, Iván Espinel y Cynthia Viteri son personas que abrazan mucho a los ciudadanos, invaden el espacio vital de cada persona y fraternizan de mejor manera. El resto de candidatos respetaron más el espacio personal y la mayoría de las veces se limitaron a un saludo cordial con la mano, como el caso de Patricio Zuquilanda y Washington Pesántez.

\section{Olfato}

En este caso, aplicándolo a los candidatos se tomó en cuenta factores como el aseo personal de cada uno, el mismo que va de la mano con la apariencia con la cual se presentaron a las diversas actividades. Todos prestaron mucha atención a su imagen y su "olor a limpio" con el cual se presentaron siempre la idea conjunta al olfato. 
El sentido del olfato va de la mano con el de la vista, un buen manejo de su imagen acompañado de una buena higiene y aseo personal dan una apariencia de pulcritud generando confianza en las personas.

\section{Gusto}

Este sentido es el más complicado de identificar en cuanto a una marca personal, debido a que es poco probable que las personas "saboreemos" a otras para poder decidir si nos agradan o no.

La interpretación del gusto se pone de manifiesto al momento de transformar las promesas en hechos y de resolver las inquietudes de las personas, periodistas o interlocutores, las dudas e incertidumbres que tienen sobre el ambiente en el que se encuentra el país y demás ítems que se manejaron durante el proceso. En esta perspectiva, el enfoque puede ir en la percepción que las personas tuvieron en lo que se denomina como el "buen sabor de boca" que les dejó su o sus candidatos, por ejemplo en los debates presidenciales que se dieron durante el periodo de la campaña electoral.

\section{Marketing Emocional}

De igual forma que los sentidos, las emociones influyen no solamente en la toma de decisiones, sino también en la percepción de un individuo o marca. Sin embargo, aunque las emociones y los sentidos están profundamente conectados, ambos son dependientes entre ellos.

Al hablar de sensaciones la referencia es hacia la sensación física u orgánica, más no emocional o sentimental, producida por un estímulo externo a través de los órganos de los sentidos. (Esguerra \& Santa Galvis, 2008). Pero sin limitarse a conceptos, sin las sensaciones no es posible producir emociones, es por esto que se dice que es una causa-efecto.

Al tener una marca emocional, es mucho más fácil conectarse con los consumidores y generar fidelidad debido a que utilizan argumentos emocionales que tocan el corazón de los clientes logrando así, conectarse más y posicionarse en su "top of heart", la base de las denominadas lovemarks. (Filser, 2003)

Debido a que las personas son seres emocionales dominados por sentimientos mucho más que por la razón, hace posible que se manejen las emociones de una manera que la persona logre asociar el consumo de un determinado producto acompañado de estados emocionales como la felicidad o el amor. (Manzano, Gavilán, \& Avelló, 2011) Tomando como referencia la importancia de los sentimientos y las emociones en el posicionamiento de una marca, es importante analizar cómo los candidatos utilizaron estos factores para poder llegar a las personas. De igual forma que una empresa quiere penetrar, permanecer y ganar participación en el mercado, los políticos deben seguir el mismo proceso para poder lograr su objetivo.

En este contexto se procede a analizar los binomios presidenciales en su forma de interactuar, su dinamismo para transmitir sensaciones y emociones.

Para Guillermo Lasso y Andrés Páez, según datos proporcionados por (CNE, 2016), en su plan de trabajo se enfocaron: impulsar la generación de empleo, promover el emprendimiento, mayor apertura comercial, fortalecer el sector productivo, ejecutar una reforma fiscal y tributaria, mayor participación de la sociedad civil y empresa privada en búsqueda de soluciones de problemas públicos y optimizar el sistema de salud (Plan de Trabajo Creo - Suma, 2017). La propuesta de Lasso se enfocó directamente hacia atacar el problema del desempleo. El mensaje que transmitió es de esperanza para las personas que se encuentran desempleadas. Es así que su propuesta de "un millón de empleos en cuatro años de gobierno" buscó calar en aquellas personas.

El plan de trabajo de Lenín Moreno y Jorge Glas se enfocó en la revolución política, económica, social, ecológica, de conocimiento y las capacidades de la juventud. (Programa de Gobierno Alianza País, 2017) Debido a que Lenín es una persona que ha luchado por los derechos de los 
discapacitados, generó un sentimiento de simpatía. Al considerar las medidas que tomó previamente en su vicepresidencia en el 2007, consiguió ganarse la confianza en las personas denotando el respeto a los derechos de todos los ciudadanos.

Al ser del partido gobernante por diez años, generó un sentimiento de estabilidad al poner siempre por delante su lema de que la revolución que se ha venido dando seguirá durante su mandato, pero de una mejor manera. También recalcó que su plan será continuar lo que tenga que continuar y corregir lo que sea necesario mejorar. Y llamó porfiados y rudos a quienes repiten que él eliminará la dolarización. Se comprometió a mantenerla para lograr la estabilidad económica. (El Comercio, 2017)

En el caso de Cynthia Viteri, su plan de trabajo se enfocó en los siguientes aspectos: económico, ambiental, político, social, seguridad y fuerza pública. (Plan de Gobierno Cambio Positivo, 2017). Puntos que se abordaron constantemente en sus discursos. Fue la candidata que en sus propuestas dio un lugar a las madres solteras, con esto buscó llegar a un porcentaje de la población nunca antes mencionado y así crear confianza y solidaridad con las mujeres. También se concentró en las personas con escasos recursos, con su propuesta de bajar la tarifa de energía eléctrica. Con esto ella buscó ganarse el cariño y aceptación de la población ecuatoriana.

En el plan de trabajo de Paco Moncayo y Monserrat Bustamante se pudo evidenciar en los puntos más importantes: afianzar un sistema económico sostenible, eficiente, inclusivo y responsable, fortalecer el aparato productivo y la generación de empleo, potenciar el desarrollo cultural y deportivo del país, garantizar la inclusión y seguridad social en el país, defender la libertad en la diversidad, impulsar una cultura de transparencia y de lucha contra la corrupción, profundizar la integración regional y las relaciones internacionales soberanas, seguridad, derechos humanos, justicia y control. (Plan de Trabajo Paco Moncayo, 2017)

Lo que se pudo denotar fue que Paco Moncayo propuso luchar por los derechos de la comunidad LGBT e inclusión dentro de la sociedad. Por otro lado, también estuvieron los jubilados del IESS a quienes prometió devolver sus jubilaciones y pagar lo que les corresponde por derecho. Al ser él un ex militar también buscó velar por sus ex compañeros, ofreciendo devolverles ciertos derechos y beneficios perdidos durante el gobierno saliente.

Con respecto a la candidatura de Abdalá Bucaram Pulley y Ramiro Aguilar, incluyeron estos puntos en su plan de trabajo: crecimiento económico y erradicación de la pobreza, bajar impuestos, eliminar impuestos, reformar el código monetario, re institucionalizar el estado, acabar la discriminación laboral al segmento de adultos mayores, rendición de cuentas en tiempo real con un programa llamado "hazlo público". (Partido Político Fuerza Ecuador Lista $10,2017)$

Abdalá Bucaram Pulley, a lo largo de su campaña presidencial, supo llegar a la gente con su sentido del humor y carisma que lo ha caracterizado, logró ganarse el cariño de la gente. Llegó al corazón de las personas con su propuesta de erradicar la pobreza y dar mano dura a la corrupción, además con su distintivo y particular baile junto a su esposa Gabriela Pazmiño, imagen de televisión reconocida a nivel nacional, sacó más de una sonrisa a varias personas, a la par de escuchar sus peticiones. También propuso incluir el lenguaje de señas en las escuelas para que así las personas con esta capacidad especial puedan ser entendidas.

En la propuesta de Iván Espinel y Doris Quiroz se evidenció lo siguiente: seguridad y justicia, empleo, productividad, desarrollo agrícola, educación para el desarrollo, gestión ambiental, tecnología e innovación, sostenibilidad y resiliencia, salud y bienestar, democracia participativa y reactivación económica. (Plan de Trabajo Fuerza Compromiso Social, 2017)

Iván Espinel, al ser el candidato más joven a la presidencia de la república, buscó calar con su mensaje señalando que la juventud no era un impedimento para llegar al poder, lo cual se 
convirtió en un punto a favor en unos casos y en contra en otros, habló sobre eliminar la corrupción, llegando con un mensaje que para el pueblo es siempre importante.

Washington Pesantez y Álex Alcívar se enfocaron en los siguientes puntos dentro de su plan de trabajo: salud, deporte, política de educación pública y cultural, turismo y cultura, transporte y movilidad, política de comunicación, seguridad, justicia, migración, energía, política internacional. (Movimiento UNIÓN ECUATORIANA lista 19, 2016)

A lo largo de su campaña se caracterizó por su presencia formal y recta, esto provocó que una parte de la población sienta confianza y lealtad hacia este candidato. A su vez, dio una apariencia de seguridad por la amplia experiencia que ha tenido a lo largo de su vida profesional, tanto en el sector público como en el privado.

Patricio Zuquilanda y Johnnie Jorgge presentaron los siguientes puntos en su plan de trabajo: sistema político, sistema económico, sistema social, sistema judicial, sistema agro productivo y sistema internacional. (Partido Sociedad Patriótica, 2017). Sin embargo, en los varios discursos se enfocó principalmente en atacar al gobierno actual y a sus competidores. Puso la mayor cantidad de sus esfuerzos en hablar sobre la corrupción y cómo pensaba erradicarla, lo cual generó empatía con todos los ciudadanos que se encuentran en contra a este tema.

\section{Conclusiones}

Es evidente que las personas, al igual que los productos, buscan formar una marca que sea sólida y aceptada por los consumidores. La combinación de un correcto manejo, tanto de los sentimientos como de las emociones, es de suma importancia para lograr el acercamiento deseado con las personas.

En la actualidad, y principalmente en la campaña del 2017, el hecho de que los políticos intentaron llegar a los electores mediante los sentidos para lograr un mayor impacto y, por ende, una recordación y posicionamiento, hizo que se eligiera minuciosamente a cuál sentido se ataque y que emoción o sentimiento se quiere que tengan las personas hacia el candidato.

El objetivo principal de los candidatos es persuadir e influir en las personas para así ganar votos y alcanzar su meta de ser el presidente/ta del Ecuador. Cada uno de los sentidos fue impulsado por las diversas acciones y actividades que cada binomio presidencial aplicó; sin embargo, el sentido al cual más se enfocaron los candidatos durante la campaña en el 2017 fue el del oído, al manejar un ataque constante a las políticas y decisiones del gobierno saliente con mensajes en contra y muchas veces desafiantes.

Por otro lado, debido al peso que tienen las redes sociales hoy en día y la influencia que se puede generar mediante éstas, el uso de un recurso auditivo como jingles o canciones especiales fue un recurso que también se lo utilizó y supo conseguir adeptos.

En la política las pasiones pesan mucho más incluso que la misma razón, el éxito de los candidatos radica en saber prender esa llama que permita que los electores adopten una marca personal política como propia y que sobre todo la consuman con su voto.

La aplicación de Marketing Sensorial en las campañas políticas y, específicamente, en el Marketing Electoral, debe saber guardar una relación de afecto y empatía entre el candidato y el elector. Llegar a empatar ambos requerimientos provocan que se vuelvan candidatos lovemarks y que eventualmente lleguen al poder, sea cual fuere el cargo por el que están postulando. 


\section{Referencias bibliográficas}

Alonso Coto, M. A., \& Adell, A. (s.f.). Marketing Político 2.0.

Bonnenmaison, J. M. (2017). Fundación Lenguaje Corporal. Obtenido de https://lenguajecorporal.org/importancia-proxemia-lenguaje-corporal/

CNE. (18 de Noviembre de 2016). Consejo Nacional Electoral. Obtenido de http://cne.gob.ec/es/institucion/sala-de-prensa/noticias/3755-ocho-binomiospresidenciales-inscritos-para-elecciones-2017

CNE. (2016). Resolución PLE-CNE-10-26-7-2016.

El Comercio. (30 de marzo de 2017). El Comercio. Lenín Moreno ofreció ejecutar su plan Toda una vida, en el cierre de su campaña electoral.

Esguerra, A., \& Santa Galvis, J. (2008). Javeriana. Obtenido de http://www.javeriana.edu.co/biblos/tesis/comunicacion/tesis106.pdf

Fara, C. (2013). Acciones para una buena comunicación en campañas electorales: manual de marketing y comunicación política. Buenos Aires: Konrad Adenauer Stiftung.

Filser, M. (2003). Le Marketing Sensoriel; la quête de líntégration théorique et managériales. Revue Française du Marketing, 5-11.

Juan de Salinas. (3 de Diciembre de 2014). Obtenido de http://www.juandesalinas.com/marketing-personal-sensorial/

La marca personal. (2016). La marca personal. Obtenido de http://www.lamarcapersonal.com/es/que-es-marca-personal

Lindstrom, M. (2007). Brand sense: Publicidad a través del imperio de los sentidos. Marketing News, 6.

Manzano, R., Gavilán, D., \& Avelló, M. y. (2011). Marketing Sensorial Comunicar con los sentidos en el punto de venta. España: Pearson.

Mehrabian, A. y. (1974). An approach to environmental psychology . Cambridge: MIT Press.

Movimiento UNIÓN ECUATORIANA lista 19. (2016). CNE. Obtenido de https://app05.cne.gob.ec/CANDIDATOS2017/GeoCandidatos.aspx

Pacheco, C., Carmona, C., \& Zapata, J. D. (2013). Marketing Sensorial Como Herramienta para captar y satisfacer al cliente. Obtenido de http://repository.urosario.edu.co/bitstream/handle/10336/4275/10305649122013.pdf? sequence $=1$

Partido Político Fuerza Ecuador Lista 10. (2017). CNE. Obtenido de https://app05.cne.gob.ec/CANDIDATOS2017/GeoCandidatos.aspx

Partido Sociedad Patriótica. (2017). Programa de Gobierno.

Pérez Ortega, A. (2008). Marca Personal: Cómo convertirse en la opción preferente. Madrir: ESIC.

Plan de Gobierno Cambio Positivo. (2017). CNE. Obtenido de https://app05.cne.gob.ec/CANDIDATOS2017/GeoCandidatos.aspx

Plan de Trabajo Creo - Suma. (2017). CNE. Obtenido de https://app05.cne.gob.ec/CANDIDATOS2017/GeoCandidatos.aspx 
Plan de Trabajo Fuerza Compromiso Social. (2017). CNE. Obtenido de https://app05.cne.gob.ec/CANDIDATOS2017/GeoCandidatos.aspx

Plan de Trabajo Paco Moncayo. (2017). CNE. Obtenido de https://app05.cne.gob.ec/CANDIDATOS2017/GeoCandidatos.aspx

Programa de Gobierno Alianza País. (2017). CNE. Obtenido de https://app05.cne.gob.ec/CANDIDATOS2017/GeoCandidatos.aspx

Programa de Gobierno del Partido Sociedad Patriótica. (2017). Obtenido de https://app05.cne.gob.ec/CANDIDATOS2017/GeoCandidatos.aspx

Robles León, L. (27 de enero de 2015). Vive Marketing Experiencial. Obtenido de https://vivemarketingexperiencial.com/tag/marca-personal-2/

Santos, F. (2013). Marketing sensorial y el imperio de los sentidos. PuroMarketing, 1-3.

Subirós Saballs, E. (29 de 05 de 2015). Universidad Internacional de la Rioja. Obtenido de http://reunir.unir.net/handle/123456789/3248

Turley, L. y. (2000). Atmospheric Effects on Shopping Behavior: A Review of the Experimental Evidence. Journal of Business Research Vol. 49(2), 193-211.

Vieira, V. (2013). "Stimuli-Organism-Response Framework: a meta-analytic review in the store environment. Journal of Business Research, Vol 66 (9), 1420-1426. 